\title{
Evaluation of Antibiotic Resistance Patterns and Frequency of Carbapenemase-Producing Acinetobacter baumannit Isolates by the Carbacineto NP Test
}

\author{
Shahram Shahraki Zahedani (PhD) \\ Department of Microbiology, School \\ of Medicine, Infectious Diseases and \\ Tropical Medicine Research Center, \\ Zahedan University of Medical \\ Sciences, Zahedan, Iran \\ Rogayeh Javadi (MSc) \\ Department of Microbiology, Faculty \\ of Medicine, Zahedan University of \\ Medical Sciences, Zahedan, Iran \\ Corresponding author: Rogayeh \\ Javadi \\ Tel: +98-5413414558 \\ E-mail: r.javadi3599@gmail.com \\ Address: Department of microbiology, \\ Faculty of Medicine, Zahedan \\ University of Medical Sciences, \\ Zahedan, Iran
}

Received : 08 Oct 2017

Revised: 03 Nov 2017

Accepted: 07 Nov 2017

Shahram Shahraki Zahedani https://orcid.org/00000003-4348-8184

\begin{abstract}
Background and Objectives: Acinetobacter baumannii is an opportunistic pathogen associated with nosocomial infections. Treatment of infections caused by this bacterium has become challenging due to increasing rate of resistance to a wide range of antibiotics such as carbapenems. One of the main mechanisms of resistance to carbapenems is the production of carbapenemase. The objective of this study was to evaluate antibiotic resistance patterns and frequency of carbapenemase-producing A. baumannii strains using the CarbAcineto NP Test.

Methods: In this descriptive cross-sectional study, 130 A. baumannii isolates were collected from clinical specimens of teaching hospitals in Zahedan in 2016. After determining the antibiotic resistance patterns, all $A$. baumannii isolates were examined using the phenotypic method of CarbAcineto NP test to evaluate production of carbapenemase enzymes.

Results: Based on the antibiogram results, more than $90 \%$ of the isolates were resistant to the antibiotics tested in this study. However, the lowest rate of resistance was observed against colistin, minocycline, tigecycline and doxycycline, respectively. Based on the results of the CarbAcineto NP test, 96\% of carbapenem-resistant strains were positive for the production of carbapenemases.

Conclusion: Due to the high resistance of A. baumannii to carbapenems, they are not currently suitable for the treatment of infections caused by this bacterium. However, since most carbapenem-resistant strains are susceptible to colistin, minocycline, tigecycline, and doxycycline, these antibiotics or their combination are recommended for the treatment of the infections caused by the resistant strains. Rapid identification of carbapenemase-producing bacteria using efficient methods such as CarbAcineto NP test is essential to prevent their spread, particularly in hospitals.
\end{abstract}

KEYWORIDS: Acinetobacter baumannii, Carbapenemase, CarbAcinetoNP Test. 


\section{INTRODUCTION}

Acinetobacter baumannii is a gramnegative, non-motile, non-fermenting, oxidasenegative, and opportunistic pathogenic coccobacillus. The bacterium can survive on solid and dry surfaces for up to 5 months, mainly because of simple nutritional requirements, ability to grow in a wide range of temperatures and $\mathrm{pH}$ values. They also have high degree of resistance to disinfectants and antiseptics, and ability to form biofilm on abiotic and biotic surfaces. A. baumannii has a natural ability to acquire antibiotic resistance mechanisms, all of which are essential for spread of hospital infections caused by the bacterium (1). The most common nosocomial infections caused by $A$. baumannii are ventilator-associated pneumonia and septicemia. They are associated with high mortality rate, as well as other infections including skin and soft tissue infections in burn patients, wound infection, urinary tract infection, and (rarely) secondary meningitis (2). The bacterium was the main cause of wound infections among military personnel in the wars in Afghanistan and Iraq, earning the nickname "Iraqibacter". In a study in the US on war-wound infections in Iraq and Afghanistan during 2007-2008, it was found that $63 \%$ of all bacterial isolates from biopsy tissues were A. baumannii (3). In 2004, the American Centers for Disease Control considered A. baumannii accountable for about $80 \%$ of all Acinetobacter infections.

Treatment of infections caused by these bacteria has become challenging, especially in recent years, due to the ability of these bacteria to obtain resistance markers (4). Carbapenems are a group of beta-lactam antibiotics with extensive activity against gram-negative and gram-positive bacteria, and are used as the last-line treatment. However, in recent years, resistance to carbapenems has increased significantly. Different mechanisms are responsible for the resistance of A. baumannii strains to carbapenems, but acquisition of carbapenemase genes is regarded as the most important mechanism (5). There are various molecular and phenotypic methods for detection of carbapenemase-producing strains. The phenotypic method, also known as the CarbAcineto NP test, is simple to use, cheap, rapid, and highly specific and sensitive method of detecting carbapenemase-producing Actinobacter spp.(6). It is essential to evaluate prevalence of carbapenemase-producing $A$. baumannii strains in different locations to determine the proper treatment options and methods for preventing the spread of infections caused by these bacteria. The objective of this study was to investigate antibiotic resistance patterns and frequency of carbapenemase-producing A. baumannii using the CarbAcineto NP test.

\section{MATERIAL AND METHODS}

In this study, 160 strains of Acinetobacter spp. were collected from teaching hospitals in Zahedan (Iran) within a 9-month period in 2016. The strains had been isolated from various clinical samples such as endotracheal tube, blood, ulcer, cerebrospinal fluid, urine and other body secretions. Among the 160 strains, 130 strains were determined as $A$. baumannii using biochemical tests and polymerase chain reaction (PCR). First, the bacteria were cultured on MacConkey agar and blood agar to obtain pure colonies. Then, the primary isolation of $A$. baumannii was carried out using multiple tests including triple sugar iron agar test, motility test in SIM medium, oxidase test (negative), growth at 42 ${ }^{\circ} \mathrm{C}$ and oxidative/fermentation glucose test (positive) (7). However, since the phenotypic methods are not able to differentiate and detect A. baumannii, the molecular method (PCR) was used for the detection of blaOXA-51 gene. PCR is a an accurate, reliable and easy method of differentiating A. baumannii species (8). For amplification of the $O X A-51$ gene, the boiling method was used for extraction of bacterial DNA. First, 24 hours after bacterial culture, a standard loop $(10 \mu \mathrm{L})$ of bacteria was removed from the culture media and transferred to a 1.5 $\mathrm{ml}$ microtube containing $150 \mu \mathrm{L}$ of normal saline. The content of the tube was mixed well until a uniform and concentrated suspension was obtained. Then, the microtube was placed in boiling water for 20 minutes and then centrifuged at $12500 \mathrm{rpm}$ for 15 minutes. Approximately, $100 \mu \mathrm{L}$ of supernatant was transferred to a $0.5 \mathrm{ml}$ microtube. Primers used for amplification of the $O X A-51$ gene are presented in Table 1.

The PCR reaction solution $(25 \mu \mathrm{L})$ contained $22.5 \mu \mathrm{L}$ of buffer mix, $0.5 \mu \mathrm{L}(10 \mathrm{pmol})$ of each primer, $0.1 \mu \mathrm{L}$ of Taq DNA polymerase and $1.5 \mu \mathrm{L}$ of DNA. In order to prepare the buffer mix, $5 \mathrm{ml}$ of deionized distilled water 
was mixed with $1 \mathrm{ml}$ of (10X buffer), $300 \mu \mathrm{L}$ of $\mathrm{MgCl}_{2}(50 \mathrm{mM})$ and $70 \mu \mathrm{L}$ of dNTP $(10 \mathrm{mM})$ and later brought to volume of $10 \mathrm{ml}$ using deionized distilled water. The buffer was distributed in $1.5 \mathrm{ml}$ microtube and then kept in freezer.

The amplification program was as follows: primary denaturation at $94{ }^{\circ} \mathrm{C}$ for 5 minutes, denaturation at $94{ }^{\circ} \mathrm{C}$ for 30 second, annealing at $53{ }^{\circ} \mathrm{C}$ for 35 seconds, extension at $72{ }^{\circ} \mathrm{C}$ for 1 minute, and 35 cycles of final extension at $72{ }^{\circ} \mathrm{C}$ for 10 minutes. PCR products were electrophoresed on $1.2 \%$ agarose gel stained with safe stain and then examined with gel doc (Figure 1). Antibiotic resistance patterns of samples containing the OXA-51 gene (130 strains of $A$. baumannii) were evaluated. For this purpose, 20 antibiotic disks and E-test were used for colistin and imipenem antibiotics. The antibiotic disks used in the study were as follows: Aztreonam $(30 \mu \mathrm{g})$, piperacillin $(100 \mu \mathrm{g})$, piperacillin tazobactam $(110 \mu \mathrm{g})$, ciprofloxacin $(5 \mu \mathrm{g})$, ceftriaxon $(30 \mu \mathrm{g})$, levofloxacin $(5 \mu \mathrm{g})$, ceftazidime $(30 \mu \mathrm{g})$, imipenem $(10 \mu \mathrm{g})$, meropenem $(10 \mu \mathrm{g})$, cefepime $(30 \mu \mathrm{g})$, cotrimoxazole $(25 \mu \mathrm{g})$, rifampin $(30 \mu \mathrm{g})$, amikacin $(30 \mu \mathrm{g})$, tobramycin $(10 \mu \mathrm{g}), \quad$ gentamicin $(120 \mu \mathrm{g}), \quad$ ampicillin sulbactam $(20 \mu \mathrm{g}), \quad$ doxycycline $(30 \mu \mathrm{g})$, minocycline $(30 \mu \mathrm{g})$ tigecycline $(15 \mu \mathrm{g})$ and colistin $(10 \mu \mathrm{g})$.

All disks were purchased from MAST Co. and standard strain of Escherichia coli ATCC 25922 was used for quality control. Antibiotic susceptibility testing was done based on Clinical and Laboratory Standards Institute (CLSI) guidelines and criteria for all antibiotics except for tigecycline (10). In this study, resistance of A. baumannii to tigecycline was evaluated based on two criteria, American Food and Drug Administration (FDA) criteria for
Enterobacteriaceae. and the Jones criterion for Acinetobacter spp. (11). First, the solutions were prepared according to the CLSI guidelines for the Carba NP Test as described below (10). Solution A: in a 25 to $50 \mathrm{ml}$ container, $16.6 \mathrm{ml}$ of deionized distilled water were mixed with $2 \mathrm{ml}$ of $0.5 \%$ phenol red solution and $180 \mu \mathrm{l}$ of $10 \mathrm{Mm} \mathrm{ZNSO}_{4}-7 \mathrm{H}_{2} \mathrm{O}$. In addition, $\mathrm{pH}$ of the solution was adjusted to 7.8 using $\mathrm{NaoH}(0.1 \mathrm{~N})$ or HCL $(10 \%)$. The solution was kept away from light and stored at $4-8{ }^{\circ} \mathrm{C}$. The solution remains stable until it is discolored. Solution B: imipenem powder was added to solution A to obtain a $6 \mathrm{mg} / \mathrm{ml}$ solution. The solution remains stable for three days in refrigerator.

Note: Phenol red $(0.5 \%)$ and zinc sulfate $(10 \mathrm{mM})$ are kept at room temperature. These two solutions are stable at room temperature for one year.

In a $1.5 \mathrm{ml}$ microtube, $100 \mu \mathrm{L}$ of $\mathrm{NaCl}$ solution (5M) was mixed with $10 \mu \mathrm{L}$ of bacteria until a uniform and concentrated suspension was achieved. Next, $100 \mu \mathrm{l}$ of solution B was added to the solution. After through mixing, the tube was incubated for two hours at $37{ }^{\circ} \mathrm{C}$. Color change from red to yellow or orange confirmed production of carbapenemase (6). Metallo- $\beta$-lactamase positive Klebsiella pneumonia (Pasteur Institute of Iran) was used as positive control, while E. coli ATCC 25922 and a blank tube (reagent without bacteria) were used as negative control.

\section{RESULTS}

Table 2 demonstrates the results of antibiotic susceptibility testing for all $130 \mathrm{~A}$. baumannii strains. Table 3 represents the results of resistance pattern and susceptibility testing for tigecycline based on the Jones and FDA criteria.

Figure 1- Image of gel electrophoresis for the $O X A-51$ gene. Column 4: positive control, column 5: negative control, column 9: Blank and columns 1, 2, 3, 6, 7, 8: strains containing the $O X A-51$ gen.

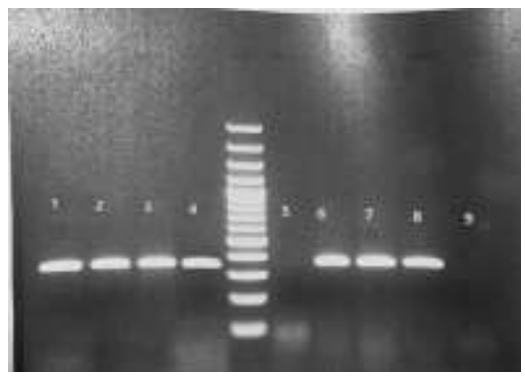


16/ Evaluation of Antibiotic. . .

Table 1- Primers used for the amplification of $O X A-51$ gene (9)

\begin{tabular}{ccl}
\hline Gene name & Nucleotide sequence & Product size \\
\hline bla oxA - 51F & TAA TGC TTT GAT CGG CCT TG & $353 \mathrm{bp}$ \\
bla oxA 51R & TGG ATT GCA CTT CAT CTT GG & \\
\hline
\end{tabular}

Table 2- Frequency of antibiotic resistance for $\boldsymbol{A}$. baumannii strains

\begin{tabular}{|c|c|c|c|}
\hline Antibiotics & Resistant & Moderate & Susceptible \\
\hline 1. Aztreonam & $125(96.2 \%)$ & $1(0.8 \%)$ & $4(3 \%)$ \\
\hline 2. Piperacillin & $125(96.2 \%)$ & 0 & $5(38 \%)$ \\
\hline 3. Piperacillin tazobactam & $124(95.4 \%)$ & $1(0.8 \%)$ & $5(38 \%)$ \\
\hline 4. Ciprofloxacin & $124(95.4 \%)$ & $\mathbf{0}$ & $6(4.6 \%)$ \\
\hline 5. Ceftriaxone & $124(95.4 \%)$ & $4(3.1 \%)$ & $2(1.5 \%)$ \\
\hline 6. Levofloxacin & $123(94.6 \%)$ & 0 & $7(5.4 \%)$ \\
\hline 7. Ceftazidime & $123(94.6 \%)$ & $\mathbf{0}$ & $7(5.4 \%)$ \\
\hline 8. Imipenem & $123(94.6 \%)$ & $\mathbf{0}$ & $7(5.4 \%)$ \\
\hline 9. Cefepime & $123(94.6 \%)$ & $1(0.8 \%)$ & $6(4.6 \%)$ \\
\hline 10. Cotrimoxazole & $121(93.1 \%)$ & $3(2.3 \%)$ & $6(4.6 \%)$ \\
\hline 11. Rifampin & $121(93.1 \%)$ & $7(5.4 \%)$ & $2(1.5 \%)$ \\
\hline 12. Meropenem & $120(92.3 \%)$ & $3(2.3 \%)$ & $7(5.4 \%)$ \\
\hline 13. Amikacin & $118(90.8 \%)$ & $5(3.8 \%)$ & $7(5.4 \%)$ \\
\hline 14. Tobromycin & $90(69.2 \%)$ & $9(6.9 \%)$ & $31(23.9 \%)$ \\
\hline 15. Gentamicin & $89(68.5 \%)$ & $11(8.5 \%)$ & $30(23 \%)$ \\
\hline 16. Ampicillin Sulbactam & $79(60.8 \%)$ & $27(20.8 \%)$ & $24(18.4 \%)$ \\
\hline 17. Doxycycline & $47(36.2 \%)$ & $\mathbf{0}$ & $83(63.8 \%)$ \\
\hline 18. Minocycline & $16(12.3 \%)$ & $8(6.2 \%)$ & $106(81.5 \%)$ \\
\hline 19. Colistin & 0 & 0 & $130(100 \%)$ \\
\hline
\end{tabular}

Table 3- Resistance patterns against tigecycline among $A$. baumannii isolates based on the Jones and FDA criteria

\begin{tabular}{cccc}
\hline Criterion & Resistant & Intermediate & Susceptible \\
\hline Jones & $\mathbf{1 4 . 3 \%}$ & $\mathbf{3 2 . 4 \%}$ & $\mathbf{5 3 . 3 \%}$ \\
FDA & $\mathbf{3 5 . 2 \%}$ & $\mathbf{4 9 . 5 \%}$ & $\mathbf{1 5 . 3 \%}$ \\
\hline
\end{tabular}

All 130 A. baumannii isolates were susceptible to colistin based on the CLSI guidelines. Seven A. baumannii strains were susceptible to imipenem. The remaining 123 strains of $A$. baumannii were resistant to imipenem. Similar findings were obtained for susceptibility to colistin and imipenem in the disk diffusion method. Based on both criteria, the lowest rate of resistance was observed against clostin, minocycline, tigecycline, doxycycline, and ampicillin-sulbactam, respectively.
In this study, all $A$. baumannii strains were evaluated by Carb AcinetoNP test.

None of the carbapenem-susceptible strains (7 strains) showed color change from red to yellow or orange, but of the 123 carbapenemresistant strains, 118 strains showed color change from red to yellow or orange, and therefore found positive for production of carbapenemase. In other words, $96 \%$ of carbapenem-resistant strains produced carbapenemase (Figure 2).

Figure 2- Image of CarbAcineto NP test results

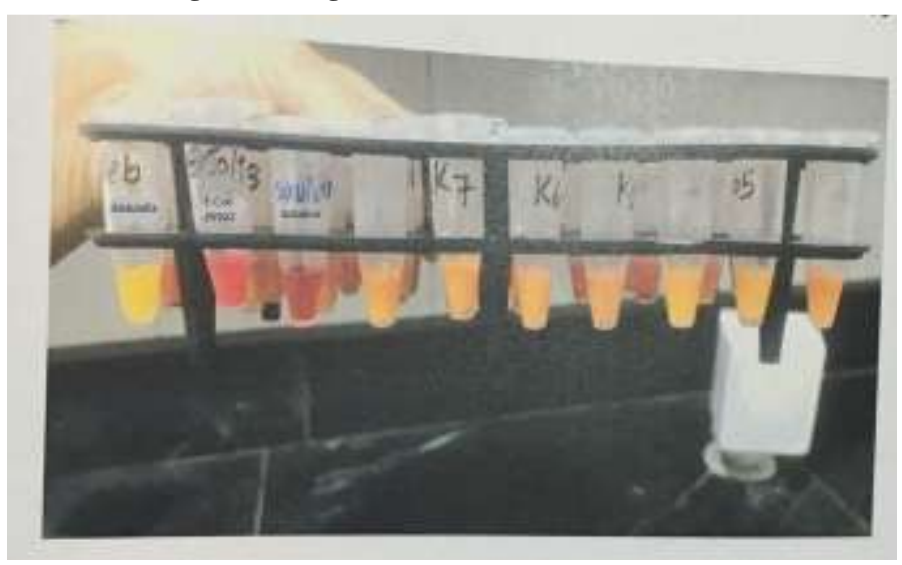




\section{DISCUSSION}

Carbapenems were drugs of choice for treatment of multidrug-resistant (MDR) $A$. baumannii infections, but the widespread use of these antibiotics in recent years has led to a rapid increase in frequency of carbapenemsresistant A. baumannii strains (12). Resistance of $A$. baumannii to carbapenems could be attributed to decreased permeability of the outer membrane following the loss or modification of porins, modification of penicillin-binding proteins (PBPs), increased expression of efflux pumps, and production of carbapenemase. Most carbapenemases identified in Acinetobacter spp. are located on mobile genetic elements that may be transferred to other clinically relevant species (6). Therefore, in order to prevent the spread of carbapenemase-producing bacteria, it is essential to separate them from strains whose carbapenem resistance is dependent on nontransferable mechanisms (loss of porines, overexpression of efflux pumps or modification of PBPs (13). Due to the intrinsic low permeability, the evaluation of carbapenemase production in Acinetobacter is more difficult compared to Enterobacteriaceae and Pseudomonas spp. Several phenotypic techniques have been proposed for the detection of carbapenemase-producing Acinetobacter spp. (14). For instance, modified Hodge test is based on the in vitro detection of carbapnemase activity, but may produce false negative results in some cases (15). Another method is the Carba NP test, which is based on colorimetry and $\mathrm{pH}$ change due to hydrolysis of the beta-lactam ring by carbapenemase. Although this test is valid for the detection of carbapenemase producers among Enterobacteriaceae and Pseudomonas spp., it has not been validated for the detection of carbapenemase-producing Acinetobacter spp., mainly because of low sensitivity (11.9\%). The CarbAcineto $\mathrm{Np}$ test was adapted from the updated version of the Carba $\mathrm{NP}$ test, and has been used as a rapid and reproducible method of detecting all carbapenemase types in Acinetobacter spp. with high sensitivity $(94.7 \%)$ and specificity $(100 \%)(6)$.

In our study, 123 A. baumannii strains (94\%) were resistant to carbapenems (imipenem and meropenem) in the CarbAcineto NP test. Of these strains, 118 strains (96\%) were found positive for the production of carbapenemase, which highlights the necessity of considering infection control criteria in hospitals. Various studies in Iran (16-19) and other countries (2023) have shown the high rate of carbapenemresistance in Acinetobacters, which indicates these drugs are no longer effective for treatment of Acinobacter infections. Another noteworthy finding was the high rate of susceptibility to minocycline. Early studies in the 1970s showed that minocycline was an effective antibiotic for the treatment of Acinetobacter infections. At that time, most $A$. baumannii strains were susceptible to more potent available antibiotics, and minocycline was not considered as the drug of choice. However, the global emergence of MDR $A$. baumannii strains led to reconsideration of old but safe therapeutic options, such as minocycline. In 2014, the CLSI re-located minocycline for Acinetobacter species in own box in test report group B. In a study by Adib Hesami et al. during 2011-2013, it was shown that minocycline is still an effective therapeutic option for the treatment of Acinetobacter infections (24). According to a study in the US in 2017, minocycline is the only available therapeutic option and active antibiotic following colistin for the treatment of infections caused by MDR A. baumannii (25). In a 6-year study, Denys et al. reported that $72 \%$ of Acinetobacter isolates were susceptible to minocycline (26). We also found that $81.9 \%$ of the isolates are susceptibility to minocycline. However, a study in China in 2017 revealed that minocycline is not commonly used in China due to toxicity. Inconsistent with the mentioned studies, a study on 2917 A. baumannii isolates in 2004-2014 showed that susceptibility to minocycline has increased in China from $13.4 \%$ to $64.5 \%$ (27)

\section{CONCLUSION}

Due to the high resistance of A. baumannii to carbapenems, they are not currently suitable for the treatment of infections caused by this bacterium. However, since most carbapenemresistant strains are susceptible to colistin, minocycline, tigecycline, and doxycycline, these antibiotics or their combination are recommended for the treatment of infections caused by the resistant strains. Rapid identification of carbapenemase-producing bacteria using efficient methods such as 
CarbAcineto NP test is essential to prevent their spread.

In addition, due to the high prevalence of carbapenemase-producing A. baumannii strains, it is crucial to consider infection control criteria in hospitals.

\section{ACKNOWLEDGEMENT}

The present article is part of a Master's

\section{REFERENCES}

1. Nowak P, Paluchowska P. Acinetobacter baumannii: biology and drug resistance-role of carbapenemases. Folia Histochemica et Cytobiologica. 2016; 54(2): 61-74. doi: 10.5603/FHC.a2016.0009.

2. Dijkshoorn L, Nemec A, Seifert H. An increasing threat in hospitals: multidrug-resistant Acinetobacter baumannii. Nature Reviews Microbiology. 2007; 5(12): 939-51. DOI:10.1038/nrmicro1789.

3. Richards A, Abu Kwaik Y, Lamont R. Code blue: Acinetobacter baumannii, a nosocomial pathogen with a role in the oral cavity. Molecular oral microbiology. 2015; 30(1): 2-15.

4. Espinal P, Marti S, Vila J. Effect of biofilm formation on the survival of Acinetobacter baumannii on dry surfaces. Journal of Hospital Infection. 2012; 80(1): 56-60. doi: 10.1016/j.jhin.2011.08.013.

5. Da Silva GJ, Domingues S. Insights on the horizontal gene transfer of carbapenemase determinants in the opportunistic pathogen Acinetobacter baumannii. Microorganisms. 2016; 4(3): pii: E29. doi: 10.3390/microorganisms4030029.

6. Dortet L, Poirel L, Errera C, Nordmann P. CarbAcineto NP test for rapid detection of carbapenemase-producing Acinetobacter spp. Journal of clinical microbiology. 2014; 52(7): 235964. doi: 10.1128/JCM.00594-14.

7. Mahon CR, Manuselis G. Textbook of diagnostic microbiology. $5^{\text {th }}$ ed. WB Saunders company; 2000.

8. Turton JF, Woodford N, Glover J, Yarde S, Kaufmann ME, Pitt TL. Identification of Acinetobacter baumannii by detection of the blaOXA-51-like carbapenemase gene intrinsic to this species. Journal of clinical microbiology. 2006; 44(8): 2974-6. doi: 10.1128/JCM.01021-06.

9. Cherkaoui A, Emonet S, Renzi G, Schrenzel J. Characteristics of multidrug-resistant Acinetobacter baumannii strains isolated in Geneva during colonization or infection. Annals of clinical microbiology and antimicrobials. 2015; 14(1): 42. doi: 10.1186/s12941-015-0103-3.

10. Jean B. Patel DA. M100 Performance Standards for Antimicrobial Susceptibility Testing. 27th Edition. Clinical and Laboratory Standards Institute. 2017; 224.

11. Liao C-H, Kung H-C, Hsu G-J, Lu P-L, Liu Y$\mathrm{C}$, Chen C-M, et al. In-vitro activity of tigecycline thesis approved and supported by the Zahedan University of Medical Sciences, Iran. The authors appreciate the support from the director and personnel of the Research Department of the university.

\section{CONFLICT OF INTEREST}

The authors declare that they have no conflict of interest.

against clinical isolates of Acinetobacter baumannii in Taiwan determined by the broth microdilution and disk diffusion methods. International journal of antimicrobial agents. 2008; 32 Suppl 3: S192-6. doi: 10.1016/S0924-8579(08)70027-X

12. Kim UJ, Kim HK, An JH, Cho SK, Park K-H, Jang H-C. Update on the epidemiology, treatment, and outcomes of carbapenem-resistant Acinetobacter infections. Chonnam medical journal. $2014 ; \quad 50(2)$ : $37-44$ doi: $10.4068 / \mathrm{cmj} .2014 .50 .2 .37$.

13. Bonnin RA, Poirel L, Nordmann P. New Delhi metallo- $\beta$-lactamase-producing Acinetobacter baumannii: a novel paradigm for spreading antibiotic resistance genes. Future microbiology. 2014; 9(1): 33-41. doi: 10.2217/fmb.13.69.

14. Bonnin RA, Nordmann P, Poirel L. Screening and deciphering antibiotic resistance in Acinetobacter baumannii: a state of the art. Expert review of anti-infective therapy. 2013;11(6):57183. doi: 10.1586/eri.13.38.

15. Bonnin RA, Naas T, Poirel L, Nordmann P. Phenotypic, biochemical, and molecular techniques for detection of metallo- $\beta$-lactamase NDM in Acinetobacter baumannii. Journal of clinical microbiology. 2012; 50(4): 1419-21. doi: 10.1128/JCM.06276-11.

16. Mohajeri P, Farahani A, Mehrabzadeh RS. Molecular Characterization of Multidrug Resistant Strains of Acinetobacter baumannii Isolated from Intensive Care Units in West of Iran. Journal of clinical and diagnostic research: JCDR. 2017; 11(2): DC20-DC22. doi: 10.7860/JCDR/2017/21156.9397.

17. Shoja S, Moosavian M, Rostami S, Farahani A, Peymani A, Ahmadi K, et al. Dissemination of carbapenem-resistant Acinetobacter baumannii in patients with burn injuries. Journal of the Chinese Medical Association. 2017; 80(4): 245-52.

18. Adibhesami H, Douraghi M, Zeraati H, Bazmi F, Rahbar M, Pourmand MR, et al. Carbapenemresistant Acinetobacter baumannii (CRAB) recovered from burn patients. Journal of Pharmacy \& Pharmaceutical Sciences. 2016; 19(3): 339-48. doi: 10.18433/J3QK6M.

19. Motamedifar M. Emergence of Multidrug Resistance and Metallo-beta-lactamase Producing Acinetobacter baumannii Isolated from Patients in Shiraz, Iran. Annals. 2016; 6(3): 162-167. doi: $10.4103 / 2141-9248.183946$. 
20. Dahdouh E, Gómez-Gil R, Pacho S, Mingorance J, Daoud Z, Suárez M. Clonality, virulence determinants, and profiles of resistance of clinical Acinetobacter baumannii isolates obtained from a Spanish hospital. PloS one. 2017; 12(4): e0176824.

10.1371/journal.pone.0176824.

21. Agarwal S, Kakati B, Khanduri S, Gupta S. Emergence of Carbapenem Resistant NonFermenting Gram-Negative Bacilli Isolated in an ICu of a Tertiary Care Hospital. Journal of clinical and diagnostic research: JCDR. 2017;11(1):DC0407. doi: 10.7860/JCDR/2017/24023.9317.

22. Kim D, Ahn JY, Lee CH, Jang SJ, Lee H, Yong $\mathrm{D}$, et al. Increasing Resistance to ExtendedSpectrum Cephalosporins, Fluoroquinolone, and Carbapenem in Gram-Negative Bacilli and the Emergence of Carbapenem Non-Susceptibility in Klebsiella pneumoniae: Analysis of Korean Antimicrobial Resistance Monitoring System. Annals of laboratory medicine. 2017; 37(3): 231-9. doi: 10.3343/alm.2017.37.3.231.

23. Warner WA, Kuang SN, Hernandez R, Chong MC, Ewing PJ, Fleischer J, et al. Molecular characterization and antimicrobial susceptibility of Acinetobacter baumannii isolates obtained from two hospital outbreaks in Los Angeles County, California, USA. BMC infectious diseases. 2016; 16(1): 194. doi: 10.1186/s12879-016-1526-y.

24. Adibhesami H, Douraghi M, Rahbar M, Abdollahi A. Minocycline activity against clinical isolates of multidrug-resistant Acinetobacter baumannii. Clinical Microbiology and Infection. 2015;21(11):e79-e80.

doi: 10.1016/j.cmi.2015.07.007.

25. Lashinsky JN, Henig O, Pogue JM, Kaye KS. Minocycline for the Treatment of Multidrug and Extensively Drug-Resistant A baumannii: A Review. Infectious diseases and therapy. 2017; 6(2): 199-211. doi: 10.1007/s40121-017-0153-2.

26. Denys GA, Callister SM, Dowzicky MJ. Antimicrobial susceptibility among gram-negative isolates collected in the USA between 2005 and 2011 as part of the Tigecycline Evaluation and Surveillance Trial (TEST). Annals of clinical microbiology and antimicrobials. 2013;12(1):24.

27. Gao L, Lyu Y, Li Y. Trends in Drug Resistance of Acinetobacter baumannii over a 10year Period: Nationwide Data from the China Surveillance of Antimicrobial Resistance Program. Chinese Medical Journal. 2017; 130(6): 659. 